\title{
Low-frequency model of breathing oscillations in Hall discharges
}

\author{
Serge Barral \\ Institute of Plasma Physics and Laser Microfusion, 01497 Warsaw, Poland \\ Eduardo Ahedo \\ E.T.S.I. Aeronáuticos, Universidad Politécnica, Madrid, 28040, Spain
}

\begin{abstract}
A paradigm for Hall discharge modeling is presented whereby only the time scale of the lowest-frequency mode is explicitly resolved. The ability of such a low-frequency model to reproduce with excellent accuracy the breathing mode is demonstrated through comparisons with a fully time-dependent numerical model. Based on this formalism, an approximate linearized model is derived which essentially constitutes a one-dimensional generalization of the classical zero-dimensional predator-prey model. The model highlights the interaction of standing plasma waves with the transport of neutral species, which involves standing and convective waves of similar magnitude. It predicts a frequency which is in close agreement with the frequency of the small perturbation modes observed in simulations. Finally, it is shown that unstable modes are in general strongly nonlinear and characterized by frequencies obeying a scaling law different from that of linear modes.
\end{abstract}

\section{INTRODUCTION}

Plasma accelerators based on Hall discharges have become a fuel-efficient alternative to conventional rocket engines for various space propulsion applications, ranging from satellite positioning to deep space probes propulsion. Hall discharges are typically realized within an annular ceramic channel in the presence of an applied radial magnetic field (Fig. 1). The electric field created between an inner anode and an external cathode drives electrons into the channel where they are partially confined by the magnetic field and ionize the working gas (most commonly xenon) introduced near the anode. The ions thus created are too heavy to be magnetized and are accelerated towards the channel exit by the axial electric field up to velocities on the order of $20 \mathrm{~km} \mathrm{~s}^{-1}$.

A worldwide surge of interest for this technology has prompted in the past 10 years a sizable intensification of theoretical and experimental studies devoted to discharge oscillations. Arguably the most ubiquitous among them are low-frequency (LF) plasma oscillations observable in the $10-30 \mathrm{kHz}$ band. They are characterized by wide ac fluctuations of the discharge current that may at times exceed the level of the dc current [1]. Although the first reports of this low-frequency mode date back from the 1970s [2], its interpretation as an ionization instability has gained wide acceptance only in the late 1990s [3,4]. Following the observation of a back-and-forth motion of the ionization front in numerical simulations $[4,5]$, these oscillations are now commonly referred to as breathing oscillations.

Despite recent attempts to explain low-frequency oscillations with semiempirical approaches [6,7], the very simple predator-prey model suggested by Fife et al. [4] remains the most favored model to interpret breathing oscillations. Its intrinsic zero-dimensional (OD) character is difficult, how- ever, to reconcile with the common view that oscillations result from a motion of the ionization front [5]. Breathing oscillations are, on the other hand, reasonably well reproduced by most time-dependent (TD) numerical models of Hall discharges [5,8-13]. Such numerical models rely, however, on complex physical descriptions that incorporate a number of unrelated time scales, making it difficult to identify the root phenomenon responsible for breathing oscillations. Following Einstein's dictum that "everything should be made as simple as possible, but no simpler," this work strives to develop a low-frequency formalism which isolates the essential time-dependent mechanisms of the breathing mode while preserving a multidimensional and physically accurate description. This formalism is applied in Sec. II to a conventional one-dimensional (1D) fluid model of Hall dis-

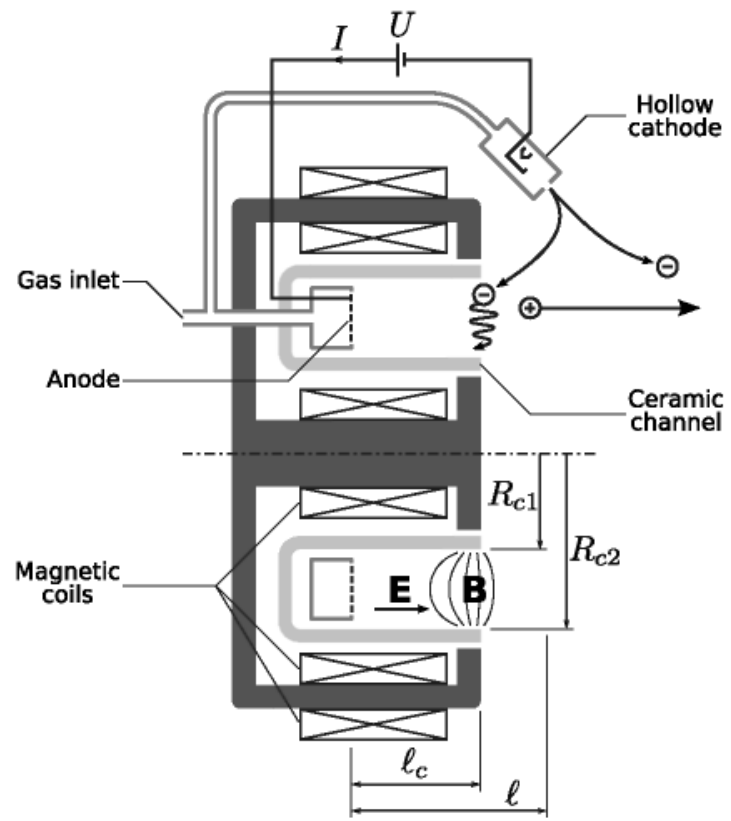

FIG. 1. Schematic of a typical Hall thruster. 
charges, and the relevance of the model thus obtained is assessed with the help of numerical simulations. A small perturbation model is subsequently derived in Sec. III, which is contrasted to the model of Fife et al. The predicted frequency is compared to the frequency of the linear modes observed in numerical simulations. Finally, nonlinear modes are briefly discussed.

\section{LOW-FREQUENCY MODEL}

\section{A. Motivation and methodology}

The breathing mode is known to involve time scales that are much longer than the characteristic relaxation and flight times of charged species [14], but comparable to the flight time of neutrals. This observation readily leads to the conjecture that for low-frequency phenomena, charged species effectively behave as if they were at any moment in a quasisteady equilibrium with a slowly evolving background of neutrals. A simplistic interpretation of this postulate might convey, however, the inaccurate view that the discharge current and plasma density reach a steady state whenever the flow of neutrals is considered frozen. In fact, for a constant discharge voltage, any sustained excess (depletion) of neutrals induces instead a steady temporal growth (decay) of the discharge current $I$ and of the plasma density $n$. This behavior follows directly from the fact that the production rate of charged species is itself proportional to the density of these species. Having this in mind, we shall argue that a quasisteady state for charged species does exist whenever the flow of neutrals is steady, although not in terms of $I$ and $n$ but in terms of quantities

$$
\nu_{I} \equiv \frac{1}{I} \frac{d I}{d t}, \quad \mathrm{n} \equiv \frac{n}{I} .
$$

Use that shall be made of this hypothesis in the derivation of a low-frequency one-dimensional fluid model proceeds as follows. Section II B introduces a conventional fully timedependent fluid description of the transport of electrons and singly charged ions. Changing then variables $I$ and $n$ for variables $\nu_{I}$ and $\mathrm{n}$, a quasisteady counterpart of the timedependent model is obtained in Sec. II C. Section II D outlines in turn a simple time-dependent model for the evolution of neutrals, which, in conjunction with the quasisteady model of charged species, eventually leads to a selfconsistent low-frequency model of the discharge. The lowfrequency model is compared in Sec. II E to a fully timedependent model by means of numerical simulations.

\section{B. Time-dependent model for charged species}

The main features of breathing oscillations highlighted by simulations appear to be largely independent from the plasma description (fluid [10], kinetic [9,15], or hybrid $[4,5,9])$ or from the consideration of physical dimensions other than the axial one (compare in particular purely axial $[5,9,10]$, axial-radial $[4,11,12]$, and axial-azimuthal [15] models). A quasineutral one-dimensional fluid formulation based on earlier works $[10,16]$ is therefore adopted, aimed at providing a reasonably accurate picture of the oscillation mechanism while remaining tractable from the perspective of mathematical analysis. Plasma magnitudes represent radially averaged values and the interaction of the plasma with the lateral walls is modeled by means of effective source terms $[10,17]$. The plasma model includes the continuity and axial momentum equations for both ions and electrons as well as the azimuthal momentum and energy equations for electrons,

$$
\begin{gathered}
\frac{\partial n}{\partial t}+\frac{\partial}{\partial x}\left(n v_{e x}\right)=\beta N n-\nu_{i w} n, \\
\frac{\partial n}{\partial t}+\frac{\partial}{\partial x}\left(n v_{i}\right)=\beta N n-\nu_{i w} n, \\
\frac{\partial}{\partial t}\left(n m_{i} v_{i}\right)+\frac{\partial}{\partial x}\left(n m_{i} v_{i}^{2}\right)=n e E+\beta N n m_{i} V-\nu_{i w} n m_{i} v_{i}, \\
e E=-\frac{1}{n} \frac{\partial}{\partial x}\left(n T_{e}\right)-\nu_{e} m_{e} v_{e x}-\omega_{c e} m_{e} v_{e \vartheta}, \\
\frac{\partial}{\partial t}\left(n v_{e \vartheta}\right)+\frac{\partial}{\partial x}\left(n v_{e x} v_{e \vartheta}\right)=\omega_{c e} n v_{e x}-\nu_{e} n v_{e \vartheta}, \\
\frac{\partial}{\partial t}\left[\frac{3}{2} n T_{e}+\frac{1}{2} n m_{e}\left(v_{e x}^{2}+v_{e \vartheta}^{2}\right)\right] \\
+\frac{\partial}{\partial x}\left[\frac{5}{2} n v_{e x} T_{e}+\frac{1}{2} n v_{e x} m_{e}\left(v_{e x}^{2}+v_{e \vartheta}^{2}\right)\right] \\
=-\beta N n \gamma_{i} \epsilon_{i}-\nu_{e w} n \epsilon_{w}-n v_{e x} e E,
\end{gathered}
$$

where $n$ is the density of electrons and singly charged ions, $N$ is the density of neutrals, $\beta$ is the ionization rate, $\nu_{i w}$ is the collision frequency for ions impacting the walls, $E$ is the axial electric field, $\omega_{c e} \equiv e B / m_{e}$ is the electron cyclotron frequency associated with the local magnetic field $B, v_{i}$ is the axial mean velocity of ions, $v_{e x}$ and $v_{e \vartheta}$ are, respectively, the axial and azimuthal mean velocities of electrons, $T_{e}$ is the temperature of electrons, $\gamma_{i} \epsilon_{i}$ is the effective ionization cost, and $m_{e}$ and $m_{i}$ refer to the mass of electrons and ions, respectively. The total momentum-transfer collision frequency for electrons, $\nu_{e}$, the electron-wall and ion-wall collision transfer frequencies $\nu_{e w}$ and $\nu_{i w}$, and the effective energy loss at the walls, $\epsilon_{w}$, are given in Appendix A as functions of the thermal and drift energies of electrons.

The model implicitly assumes that the cross section $A$ of the discharge column is constant. Subtracting Eq. (2) from Eq. (3), it can be noted that the total current through the channel,

$$
I \equiv e \operatorname{An}\left(v_{i}-v_{e x}\right)
$$

is independent of $x$. Eliminating the electric field using Ohm's law (5), the plasma equations are recast into a system of equations of the form

$$
\frac{\partial n}{\partial t}+\frac{\partial}{\partial x}\left(n v_{i}\right)=n\left(\beta N-\nu_{i w}\right)=n h_{1},
$$




$$
\begin{gathered}
\frac{\partial}{\partial t}\left(n m_{i} v_{i}\right)+\frac{\partial}{\partial x}\left(n m_{i} v_{i}^{2}+n T_{e}\right)=n h_{2}, \\
\frac{\partial}{\partial t}\left(n v_{e \vartheta}\right)+\frac{\partial}{\partial x}\left(n v_{e x} v_{e \vartheta}\right)=n h_{3}, \\
\frac{\partial}{\partial t}\left(\frac{T_{e}^{3 / 2}}{n}\right)+v_{e x} \frac{\partial}{\partial x}\left(\frac{T_{e}^{3 / 2}}{n}\right)=\frac{1}{n} h_{4} .
\end{gathered}
$$

Integrating Eq. (5), the discharge potential satisfies

$$
U \equiv \int_{x=0}^{x=\ell} E d x=\int_{x=0}^{x=\ell}\left[-\frac{1}{e n} \frac{\partial\left(n T_{e}\right)}{\partial x}+h_{5}\right] d x,
$$

where all $h_{k}$ terms $(k=1, \ldots, 5)$ are such that $\partial h_{k} / \partial n=0$, i.e., are functions of $x, N, v_{i}, v_{e x}, v_{e \vartheta}$ and $T_{e}$ only. Note that Eq. (13) implicitly neglects the potential across the anode sheath, which is much smaller than the discharge voltage and is found to play no significant role in the analysis to come.

The above equations are complemented by the boundary conditions

$$
\begin{gathered}
\left.v_{i}\right|_{x=0}=-\sqrt{\frac{5}{3} \frac{\left.T_{e}\right|_{x=0}}{m_{i}}}, \\
\left.v_{e \vartheta}\right|_{x=\ell}=\left.\frac{v_{e x} \omega_{B}}{\nu_{e}+\beta N-\nu_{i w}}\right|_{x=\ell}, \\
\left.T_{e}\right|_{x=\ell}=\text { const, }
\end{gathered}
$$

where positions $x=0$ and $x=\ell$, respectively, refer to the locations of the anode sheath edge and of the virtual cathode. Equation (14) is the Bohm condition for ions at the anode sheath edge. Boundary condition (15) for $v_{e \vartheta}$ derives from the usual local approximation where inertial terms are ignored [16]. Although the problem could be formally closed by assuming a constant current source $(I=\mathrm{const})$, a more realistic constant voltage boundary condition is assumed,

$$
U=\text { const. }
$$

\section{Quasisteady model for charged species}

The derivation of a quasisteady model based on Eqs. (3)-(7) requires special care. Indeed, dropping altogether the time derivatives in these equations fails to provide a consistent model: it is then easily seen that if a steady solution existed with $I=I_{0}$ and $n=n_{0}$, then an infinite number of valid steady solutions could be generated by simultaneously varying $I$ and $n$ proportionally to $I_{0}$ and $n_{0}$. The root of this problem lies in the incorrect assumption that $I$ and $n$ reach a steady state when the density of neutrals, $N$, is an arbitrary input: it actually turns out that a well-defined steady state exists only for the ratio $n / I$ and for the growth rate of $I$. Introducing thus variables $\nu_{I}$ and $\mathrm{n}$ defined by Eq. (1) into Eqs. (8)-(13) and subsequently dropping all explicit time derivatives, the following quasisteady model is obtained:

$$
e A n\left(v_{i}-v_{e x}\right)=1,
$$

$$
\begin{gathered}
\frac{\partial}{\partial x}\left(\mathrm{n} v_{i}\right)=\mathrm{n} h_{1}-\mathrm{n} \nu_{I}, \\
\frac{\partial}{\partial x}\left(\mathrm{n} m_{i} v_{i}^{2}+\mathrm{n} T_{e}\right)=\mathrm{n} h_{2}-\mathrm{n} m_{i} v_{i} \nu_{I}, \\
\frac{\partial}{\partial x}\left(\mathrm{n} v_{e x} v_{e \vartheta}\right)=\mathrm{n} h_{3}-\mathrm{n} v_{e \vartheta} \nu_{I}, \\
v_{e x} \frac{\partial}{\partial x}\left(\frac{T_{e}^{3 / 2}}{\mathrm{n}}\right)=\frac{1}{\mathrm{n}} h_{4}+\frac{T_{e}^{3 / 2}}{\mathrm{n}} \nu_{I}, \\
U=\int_{x=0}^{x=\ell}\left[-\frac{1}{e \mathrm{n}} \frac{\partial\left(\mathrm{n} T_{e}\right)}{\partial x}+h_{5}\right] d x,
\end{gathered}
$$

while the expressions of boundary conditions (14)-(17) remain unchanged. Unlike the steady-state form of Eqs. (8)-(17), the present system is well posed if $N(t, x)$ is an arbitrary input. Neither $I$ nor $n$ appears explicitly in the present model, which shows that the low-frequency solution is instead determined in terms of $\nu_{I}$ and $\mathrm{n}$.

\section{Transport of neutrals}

Following Refs. [5,9], the dynamics of the neutral gas is described by a simple advection model, assuming that the mean axial velocity of neutrals is constant and equal to the thermal velocity at the gas feed,

$$
V=\text { const. }
$$

This assumption could be improved upon by including the effect of diffusion at the walls, the apparent acceleration inherent to the preferential depletion of low-velocity neutrals by ionization, and the incomplete thermal accommodation of ions neutralized at the walls. The first two effects may be responsible for an increase in the mean velocity on the order of $50 \%$ between the gas feed and the end of the ionization zone [18]. All three phenomena are accounted for in twodimensional (2D) hybrid models $[8,11,12]$ and can be effectively introduced into one-dimensional models $[10,15]$. Since they do not appear to qualitatively affect the breathing mode mechanism displayed by models based on simple advection transport [5], these effects are neglected in the present study for the sake of simplicity. The transport of neutrals therefore reduces to a single continuity equation,

$$
\frac{\partial N}{\partial t}+V \frac{\partial N}{\partial x}=-\beta N n+\nu_{i w} n .
$$

The density of neutrals at the anode $(x=0)$ is mainly set by the flux of injected propellant with a small contribution from ions recombined on the anode surface, leading to the boundary condition

$$
\left.N\right|_{x=0}=\frac{1}{V}\left(\frac{\dot{M}}{A m_{i}}-\left.\left(n v_{i}\right)\right|_{x=0}\right),
$$

where $\dot{M}$ is the mass flow rate of propellant injected through the anode. 


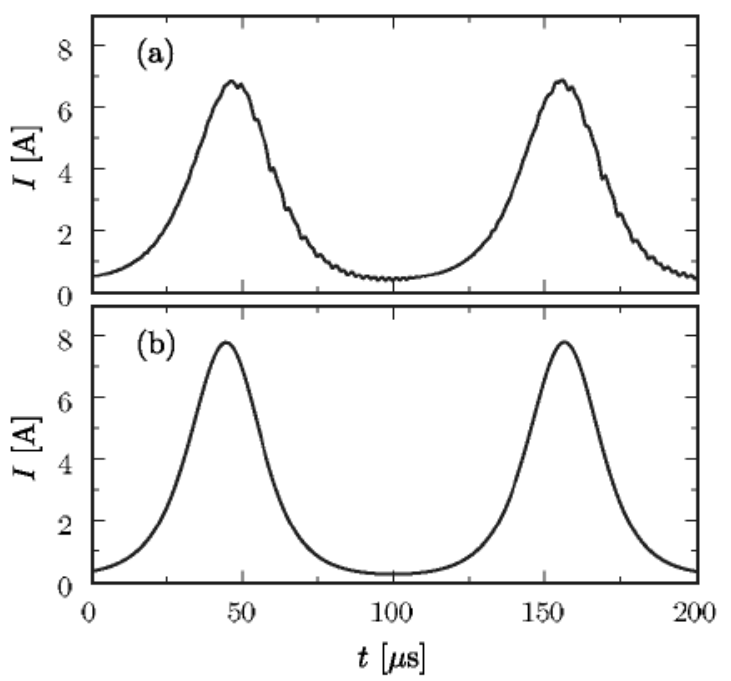

FIG. 2. Evolution of the discharge current $I$. Solutions for $U$ $=180 \mathrm{~V}$ and $B_{\max }=22 \mathrm{mT}$ using (a) the fully time-dependent model and (b) the low-frequency model. Ion transit-time oscillations can be distinguished on subfigure (a), superimposed on the low-frequency signal.

\section{E. Numerical study of the low-frequency model}

The fully TD and LF models are readily obtained by coupling the model for neutrals of Sec. II D with, respectively, the model for charged particles of Sec. II B and its quasisteady counterpart of Sec. II C. The details of their numerical implementation is deferred to Appendix B. These two models are compared for the case $U=180 \mathrm{~V}$ and $B_{\max }=22 \mathrm{mT}$ where the amplitude of oscillations is moderate enough to clearly distinguish the important features of the breathing mode; the quantitative agreement between the two models remains similar, however, notwithstanding the amplitude of oscillations.

The low-frequency behavior of the TD and LF models shown on Figs. 2-5 appear almost identical in terms of amplitude and frequency and are qualitatively very similar to earlier results of the literature $[5,10]$. Consistent with the simulations of Boeuf et al. and with experimental results [19], the breathing mode affects mostly $I$ (Fig. 2) and $n$ which exhibits a near-standing-wave pattern in phase with $I$ (Fig. 4). To a lesser extent, low-frequency oscillations also affect $N, T_{e}, v_{i}, v_{e x}$, and $v_{e \vartheta}$. The plots of $N$ on Fig. 3 highlight the characteristic back-and-forth motion of the ionization zone described earlier in simulations [5].

Self-excited oscillations are observed only within a certain parametric range of the discharge voltage and magnetic field. It is worth mentioning that no self-excited oscillations are observed with an ideal current source ( $I=$ const). The sensitivity of voltage stability thresholds toward various parameters of the model, in particular toward Bohm-type anomalous conductivity coefficients, unfortunately prevents meaningful comparisons with experiments in this regard. This is congruent with the mitigated success of former attempts at recovering the oscillation regions observed experimentally $[10,11,20]$. Incidentally, recent numerical simulations suggest that replacing the usual boundary condition $U$
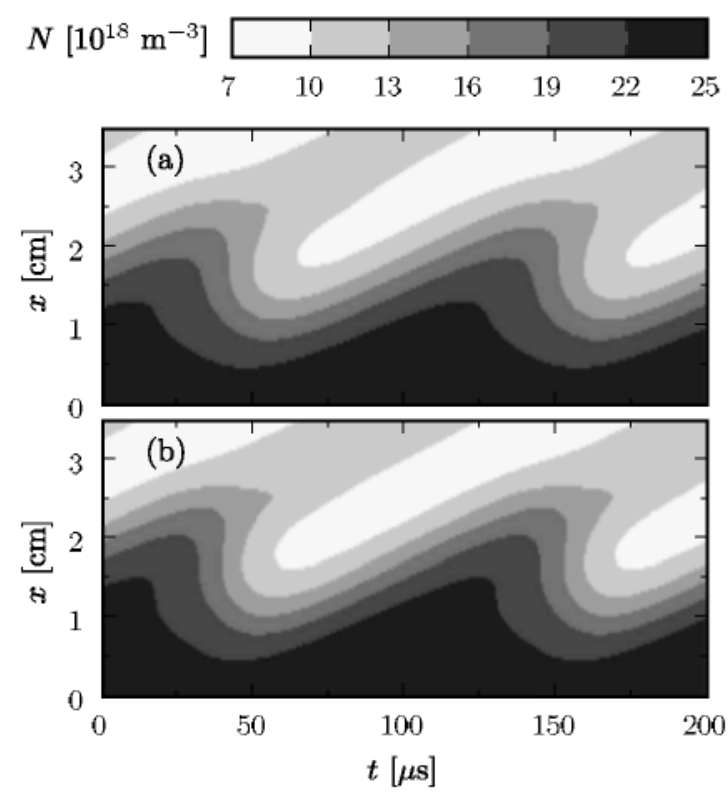

FIG. 3. Evolution of the density of neutrals $N$ in the conditions of Fig. 2. Solutions for (a) the fully time-dependent model and (b) the low-frequency model.

= const with a more realistic model of power supply strongly modifies the stability thresholds [21,22].

For the TD model, Figs 2(a), 4(a), and 5(a) show fast oscillations in the range of several hundreds of $\mathrm{kHz}$ superimposed on the low-frequency signal. They affect primarily $n, T_{e}, v_{i}, v_{e x}, v_{e \vartheta}$ and to a lower extent $I$, but are too fast to induce observable changes in the density of neutrals, as witnessed by Fig. 3(a). The propagation velocity of these oscillations lies very close to the velocity of ions, which leaves little doubt as to their relationship to the so-called ion transit
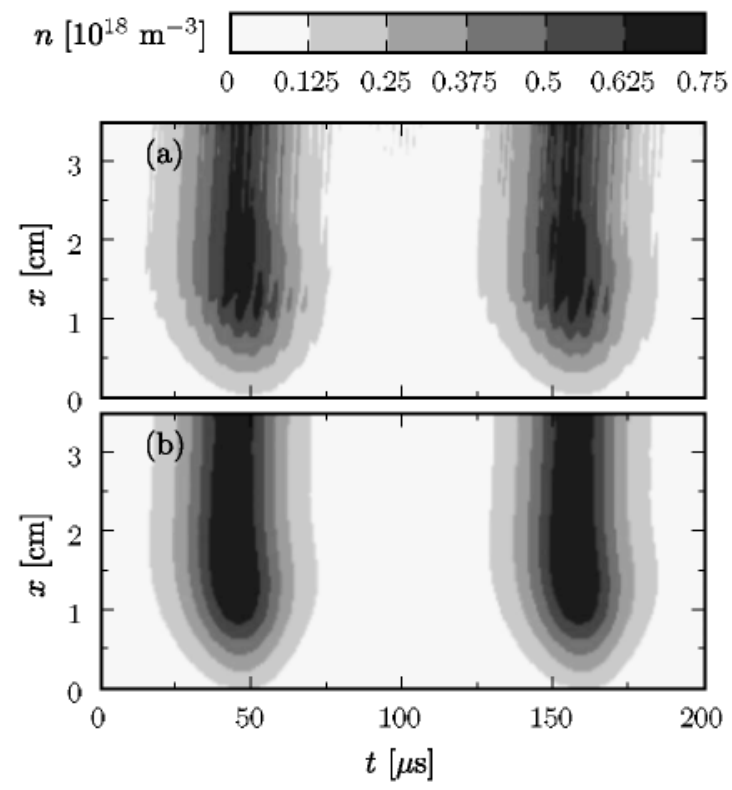

FIG. 4. Evolution of the plasma density $n$ in the conditions of Fig. 2. Solutions for (a) the fully time-dependent model and (b) the low-frequency model. Note the presence of ion transit-time waves on subfigure (a). 

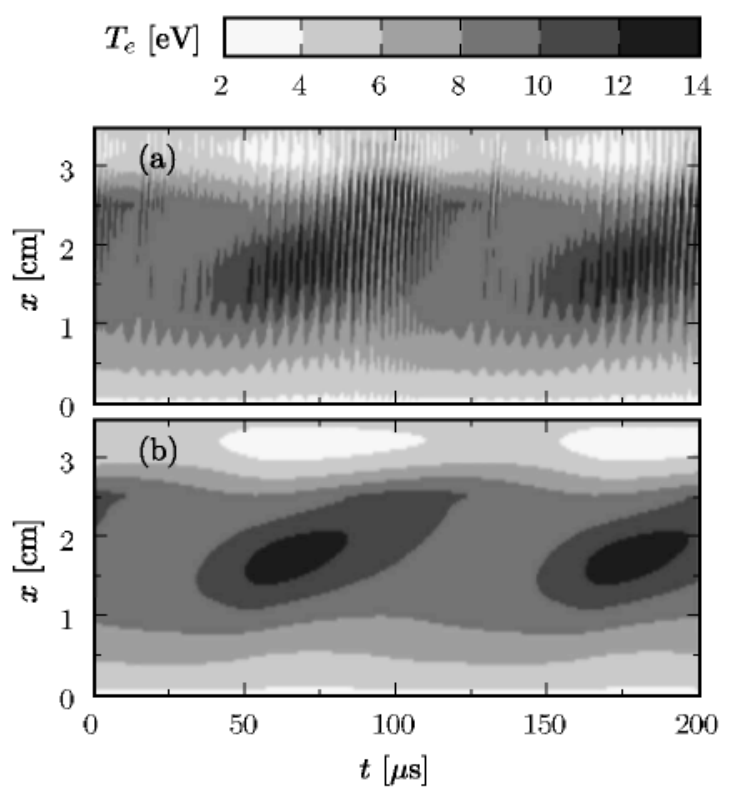

FIG. 5. Evolution of the temperature of electrons $T_{e}$ in the conditions of Fig. 3. Solutions for (a) the fully time-dependent model and (b) the low-frequency model. Note the presence of ion transittime waves on subfigure (a).

instability $[23,24]$. These fast oscillations are markedly absent in the LF model.

In conclusion, the LF approximation can be viewed as a mean to effectively isolate low-frequency phenomena from extraneous fast scale phenomena. The conceptual simplicity of the low-frequency approximation can be fully measured by noting that the plasma state in the LF model is at any moment solely determined by the instantaneous values of $I$ and $N$, which are the only time-dependent quantities explicitly resolved. This is in stark contrast to the TD model where $N, n, v_{i}, v_{e \theta}$, and $T_{e}$ are all governed by time-dependent equations.

\section{THEORETICAL ANALYSIS}

\section{A. Functional form of the low-frequency model}

By virtue of Eq. (1), the continuity equation (25) can be rewritten as

$$
\frac{\partial N}{\partial t}+V \frac{\partial N}{\partial x}=-I\left(\beta N \mathrm{n}-\nu_{i w} \mathrm{n}\right)
$$

Since the quasisteady state for charged species $\left(\mathbf{n}, v_{i}, v_{e \vartheta}, T_{e}, \ldots\right)$ obtainable from Eqs. (18)-(23) and (14)(16) can be fully determined from $\nu_{I}$ and $N$ and from the boundary conditions, Eq. (27) can be in turn written formally as

$$
\frac{\partial N}{\partial t}+V \frac{\partial N}{\partial x}=-I \mathcal{S}\left(\nu_{l}, N\right),
$$

where $\mathcal{S}$ is an operator acting on $\nu_{I} \in \mathbb{R}$ and $N:[0, \ell] \rightarrow \mathbb{R}$; in other words, ion production at a given time $t_{0}$ and location $x_{0}$ is proportional to the instantaneous current $I\left(t_{0}\right)$ and to a term that depends only on the instantaneous growth rate of the current $\nu_{I}\left(t_{0}\right)$ and on the instantaneous profile $N\left(t_{0}, x\right)$ within the whole domain. Likewise, an alternative expression of the discharge potential equation (23) can be obtained as

$$
U=\mathcal{U}\left(\nu_{l}, N\right),
$$

where $\mathcal{U}$ is a functional acting on $\nu_{I} \in \mathbb{R}$ and $N:[0, \ell] \rightarrow \mathbb{R}$. Accounting then for boundary condition $U=$ const, it becomes obvious from Eq. (29) that in order to keep the discharge voltage constant, changes in $N$ are accommodated at any instant by $\nu_{I} \equiv d \ln I / d t$. This is an important finding that contradicts the common view that these changes are accommodated by $I$ itself.

Equations (28) and (29) constitute a faithful generalization of Eqs. (14)-(23) and (25). In order to close this equation system, a slight simplification is introduced to Eq. (26) where the contribution of ions recombined at the anode is ignored, leading to the boundary condition

$$
\left.N\right|_{x=0}=\text { const. }
$$

\section{B. Linear modes}

\section{Theoretical model}

Appendix C carries out a linear mode analysis of Eqs. (28)-(30) by considering small perturbations around the steady state. The system hence obtained is expanded in terms of the small parameter

$$
\varepsilon=\frac{n_{0}}{N_{0}}
$$

with $n_{0}$ and $N_{0}$ characteristic values of the steady-state densities; in practice, $\varepsilon=\mathcal{O}\left(10^{-2}\right)$. For $\varepsilon \rightarrow 0$, the solution of the linear system raises a simple approximation of the modal frequency

$$
\omega_{0}=\sqrt{\int_{0}^{\ell} \gamma(x) \Psi(x) d x},
$$

where $\gamma(x)$ is the effective steady-state ionization frequency, which is defined in terms of the steady-state density of neutrals $\bar{N}$ as

$$
\gamma \equiv \frac{V}{\bar{N}} \frac{d \bar{N}}{d x} .
$$

Function $\Psi(x)$ can be viewed as a weighting function for the impact of a small relative perturbation $\delta N / \bar{N}$ from the steady state on the instantaneous growth rate of $I$,

$$
\frac{1}{I} \frac{d I}{d t}=\int_{0}^{\ell} \Psi \frac{\delta N}{\bar{N}} d x
$$

It can be shown that $\Psi$ exists and is uniquely defined, but no analytical expression of $\Psi$ can be given in the general case. For illustration purposes, function $\Psi$, obtained from numerical computations in the conditions of Sec. II E, is plotted together with $\gamma$ on Fig. 6. 


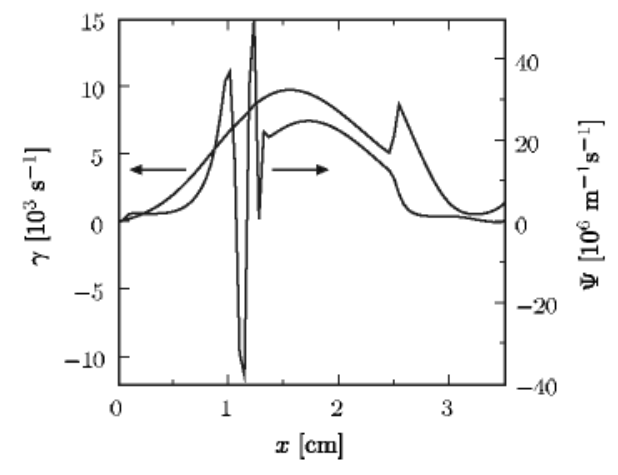

FIG. 6. Functions $\gamma$ and $\Psi$ determined numerically in the conditions of Figs. 2-5. The oscillations of $\Psi$ in the neighborhood of the sonic point $(x \approx 1 \mathrm{~cm})$ are believed to constitute a numerical artifact.

Let us now examine the orders of magnitudes of the different plasma parameters. Appendix $\mathrm{C}$ shows that the angular frequency of Eq. (32) is of magnitude

$$
\omega_{0}=\frac{V}{\ell} \mathcal{O}\left(\varepsilon^{-1 / 2}\right),
$$

which suggests that it grows with the root of the ratio of gas density to plasma density. Furthermore, it can be established that the perturbation of the plasma density is, at the leading order, a standing wave synchronized with current oscillations,

$$
\frac{\delta n}{\bar{n}} \approx \frac{\delta I}{\bar{I}},
$$

which is in agreement with the results of Figs. 2 and 4 and with earlier studies $[5,11]$. In contrast, the leading-order perturbation of the gas density consists of a standing wave with a quarter-cycle delay with respect to $\delta I$, superimposed on a traveling wave of the same order of magnitude,

$$
\delta N \approx J \frac{\delta I}{\omega}\left[\gamma(x) \bar{N}(x)-\gamma(0) \bar{N}(0) \exp \left(-J \omega \frac{x}{V}\right)\right] .
$$

This is consistent with the view that oscillations are accompanied by a motion of the ionization front. Finally, the relative perturbation of $N$ is found to be of lesser magnitude than that of $n$ or $I$,

$$
\left|\frac{\delta N}{\bar{N}}\right|=\left|\frac{\delta I}{\bar{I}}\right| \mathcal{O}(\varepsilon),
$$

which was clearly apparent in simulations (compare Figs. 2 and 4 with Fig. 3).

\section{Comparison of the predicted frequency with simulations}

The linear modes of the low-frequency numerical model are easily obtained by observing the short-term evolution of the system when starting simulations from a slightly perturbed steady state. The low-frequency model responds then as a damped or excited harmonic oscillator and $\omega$ can be directly inferred by matching the oscillation pattern of $I(t)$ to the ideal response

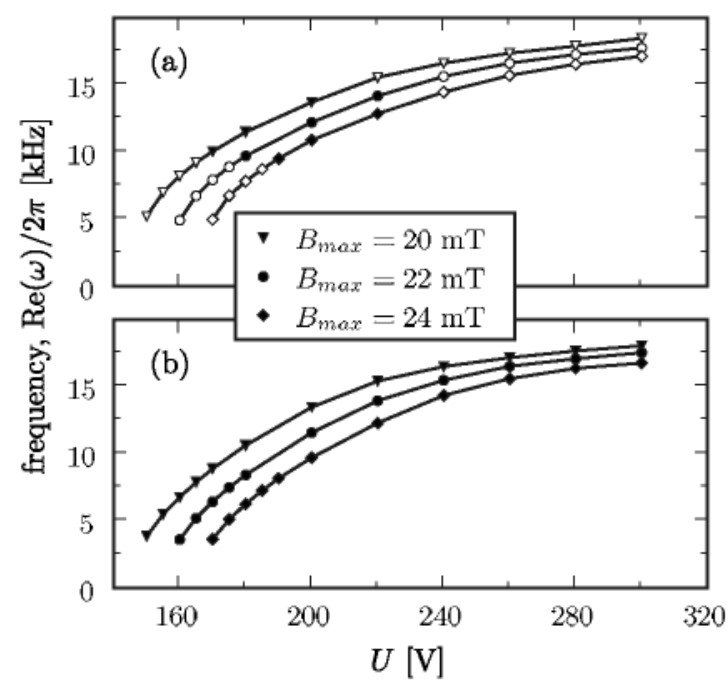

FIG. 7. Comparison between (a) the frequency of small perturbation modes of the low-frequency simulation and (b) the prediction from the linear approximation [Eq. (32)]. Linearly unstable modes $[\operatorname{Im}(\omega)<0]$ are emphasized with black markers on subfigure (a).

$$
I(t) \approx \bar{I}+\alpha \exp [\operatorname{Im}(\omega) t] \cos [\operatorname{Re}(\omega) t]
$$

In order to compare to the theoretical frequency predicted by Eq. (32), functions $\gamma$ and $\Psi$ must be determined numerically for each operating point investigated. Function $\gamma$ is directly obtained from Eq. (33), based on the steady state computed by the low-frequency model. Function $\Psi$ is computed by taking the steady state as initial condition for the simulation, with a pulse perturbation of $N$ at position $x$ where $\Psi$ is to be computed; the ensuing current growth rate is then used to determine $\Psi(x)$.

The frequency of the small amplitude modes of the simulation and the prediction of Eq. (32) are compared on Fig. 7 for various values of discharge potential and applied magnetic field. The agreement between the two is in general excellent, which provides strong support for the expansion in $\varepsilon \equiv n_{0} / N_{0}$ upon which Eq. (32) relies.

\section{Predator-prey interpretation of linear modes}

It is interesting to compare this one-dimensional treatment of the breathing mode with the linearized zero-dimensional predator-prey model of Fife et al. [4]. This latter model analyzes the temporal cycles of growth and decay of the spatially averaged gas and plasma densities, $\langle N\rangle$ and $\langle n\rangle$. The model yields marginally stable ionization oscillations of frequency

$$
\omega_{\mathrm{pp}}=\sqrt{\frac{\langle i n\rangle}{\langle\bar{N}\rangle\langle\bar{n}\rangle}},
$$

where $\langle\dot{n}\rangle$ is the steady-state ion production rate. In Hall thrusters, the relation $\dot{n} \sim N V / \ell$ holds and the following order of magnitude can thus be deduced: 


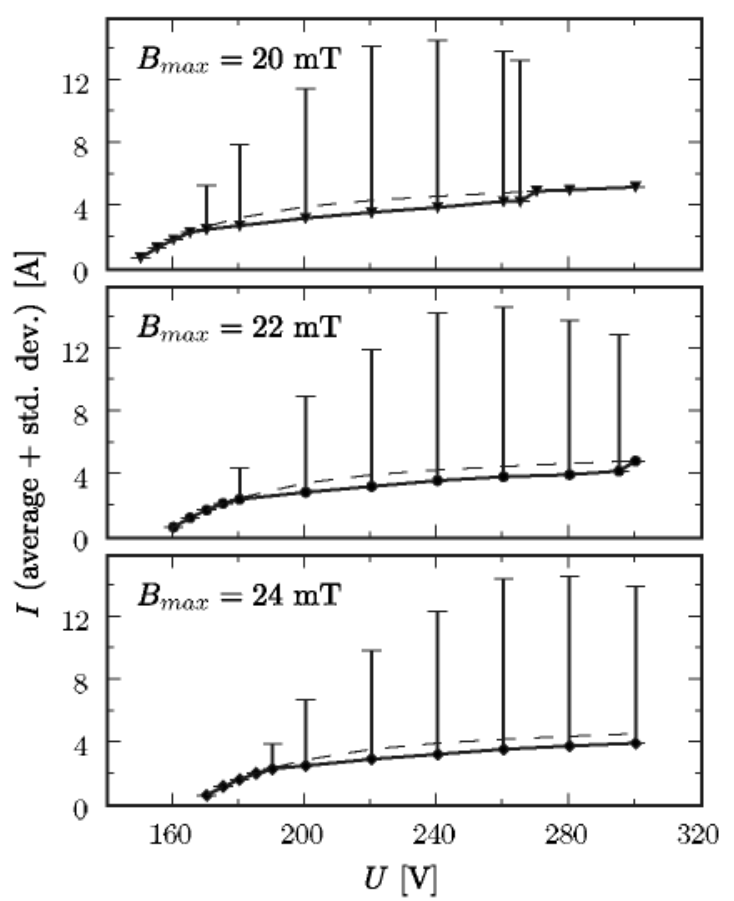

FIG. 8. Average values (solid lines) and standard deviations (vertical bars) of the discharge current for the periodic modes of the low-frequency simulation. The steady-state discharge current is plotted with a dashed line.

$$
\omega_{\mathrm{pp}}=\mathcal{O}\left(\frac{V}{\ell} \sqrt{\frac{\langle\bar{N}\rangle}{\langle\bar{n}\rangle}}\right),
$$

which coincides qualitatively with Eq. (35).

The coincidence of the frequency scaling may appear somewhat surprising at first, considering the incapacity of the zero-dimensional model to reproduce the traveling wave component of $\delta N$ in Eq. (37). The reason for this is that, even though the amplitude of the convective wave is comparable to that of the standing wave, the overall contribution of the convective wave to the growth rate of the discharge current nearly cancels in the integral of Eq. (34) owing to its relatively short wavelength.

\section{Nonlinear modes}

In both simulations and experiments, the saturated breathing instability appears as a nearly periodic signal (i.e., a stable limit cycle). Nonlinearities are thus to be expected, which will modify the frequency compared to that obtained with the small perturbation approach. In many cases, periodic solutions can be found even though the mode is linearly stable, provided that the initial conditions are far enough from the steady state. This can be observed by comparing the linearly unstable region in Fig. 7(a) to the regions where periodic solutions are observed in simulations, shown in Fig. 8. Periodic solutions typically arise from the lower threshold voltage, but remain alive beyond the upper threshold voltage for linear stability.

The detailed theory of periodic modes will be the subject of a separate work; we shall only reproduce here an approxi-

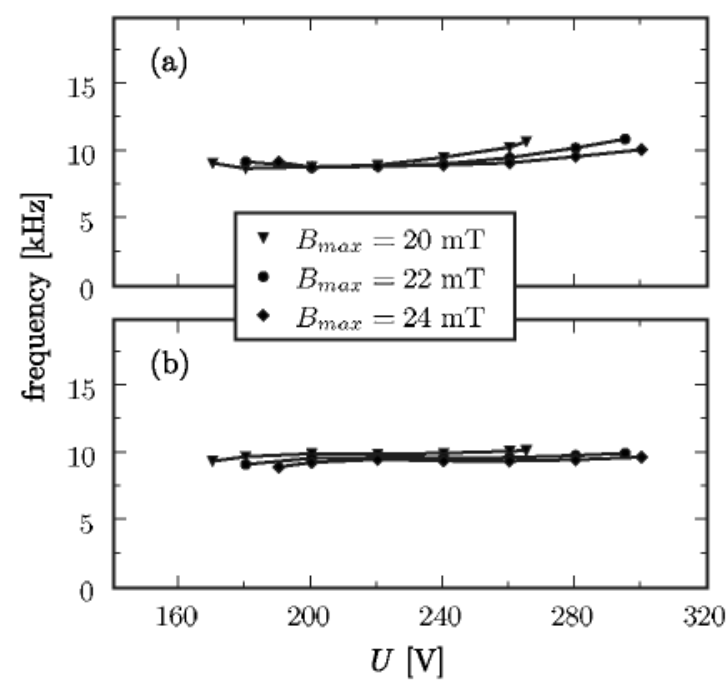

FIG. 9. Comparison between (a) the frequency of periodic solutions of the low-frequency simulation and (b) the prediction of Eq. (41) for highly nonlinear modes [25].

mation of the theoretical frequency of highly nonlinear modes [25],

$$
f \approx V \frac{\int_{0}^{\ell} \Psi(x) \gamma(x) d x}{2 \int_{0}^{\ell} \Psi(x) \int_{0}^{x} \gamma\left(x^{\prime}\right) d x^{\prime} d x},
$$

where $\gamma$ and $\Psi$ are the quantities introduced earlier in Sec. III B 1. Noting that the inverse of the ratio of integrals defines a characteristic length, it can be expected that the frequency of nonlinear modes scales proportionally to the transit frequency of neutrals.

The corresponding frequency is compared in Fig. 9 to the frequency of the periodic solutions obtained in simulations. We observe that the frequency of saturated modes significantly differs from that of linear modes, except close to the lower stability threshold where oscillation levels are moderate and nonlinearities are presumably small. Just as in the case of linear modes, the theoretical prediction is in very good agreement with numerical simulations and correctly predicts that the frequency of periodic solutions remains fairly constant when the discharge voltage and magnetic field are changed. This finding is consistent with the observation in other simulations that the frequency seems related to the transit time for neutrals [5].

\section{CONCLUSION}

Using the low-frequency paradigm and an asymptotic approach based on the parameter $\varepsilon \equiv n_{0} / N_{0}$, a general picture of the breathing mode for small perturbations has been outlined. It is shown that at the leading order, the plasma density is a standing wave while the transport of neutrals involves both a standing wave and a convective wave. However, because the convective wave changes sign within the $1 \mathrm{D}$ domain, its instantaneous effect on ionization is dominated by 
the standing-wave component and the frequency scaling of linear modes is indeed that suggested by the OD model of Fife et al. [4].

While this scaling is in excellent agreement with the small perturbation modes found in simulations, it is no longer adequate when it comes to the actual frequency of unstable modes (i.e., the saturated periodic solutions). The frequency of these nonlinear modes is largely independent from the discharge voltage and magnetic field and is lower than that of linear modes. Instead, it appears to scale proportionally to the transit frequency of neutrals, as suggested by Boeuf and Garrigues [5]. A theory of such nonlinear modes based on the low-frequency paradigm shall be developed in a separate work [25].

\section{ACKNOWLEDGMENTS}

S.B. performed this work within the frame of the French Research Group "Propulsion par Plasma dans l'Espace" (GDR 3161 CNRS/CNES/SNECMA/Universités). E.A. was supported by the Ministerio de Ciencia e Innovación of Spain (Project No. ESP2007-62694 "Motores espaciales de plasma y efecto Hall").

\section{APPENDIX A: PARAMETERS OF THE SIMULATION}

The effective frequency for ion recombination at the walls, the frequency for momentum transfer of electrons at the walls, the radial sheath potential, and net electron energy loss at the walls are given by a simplified theory of the radial sheath and presheath $[10,26]$,

$$
\begin{gathered}
\nu_{i w}=\left\{\begin{array}{cc}
\frac{4}{3} \frac{1}{R_{c 2}-R_{c 1}} \sqrt{\frac{T_{e}}{m_{i}}} \text { for } x \leq \ell_{c}, \\
0 \quad \text { for } x>\ell_{c}
\end{array}\right. \\
\nu_{e w}=\frac{\nu_{i w}}{1-\sigma}, \\
\Phi_{w}=\frac{T_{e}}{e} \log \left[\sqrt{\frac{m_{i}}{2 \pi m_{e}}}(1-\sigma)\right], \\
\epsilon_{w}=2 T_{e}+\frac{1}{2} m_{e}\left(v_{e x}^{2}+v_{e \theta}^{2}\right)+(1-\sigma) e \Phi_{w},
\end{gathered}
$$

where in our simulations, $\ell_{c}=2.5 \mathrm{~cm}$ is the length of the channel, $R_{c 1}=3 \mathrm{~cm}$ and $R_{c 2}=5 \mathrm{~cm}$ are the inner and outer channel radii, and $\sigma$ is the effective electron secondary emission yield from the walls. Assuming a linear dependence of the secondary emission to the energy of impacting electrons and taking into account a possible space charge saturation of the sheath for a xenon plasma [27], the expression for the effective secondary emission yield reads

$$
\sigma=\min \left(\frac{2 T_{e}}{\varepsilon^{*}}, 0.986\right),
$$

where the energy corresponding to a unity yield is set to $\varepsilon^{*}$ $=50 \mathrm{eV}$, which lies within the typical range for boron nitride based insulators.
The total momentum-transfer frequency for electrons is in turn

$$
\nu_{e}=\beta^{(m)} N+\alpha_{B} \omega_{c e}+\nu_{e w},
$$

where $\beta^{(m)}=2.5 \times 10^{-13} \mathrm{~m}^{3} \mathrm{~s}^{-1}$ is the assumed momentum transfer rate for electron-neutral collisions in xenon and $\alpha_{B}$ $=1 / 160$ stands for the coefficient of Bohm-type electron diffusion.

The single ionization cost for xenon is set to $\epsilon_{i}$ $=12.1 \mathrm{eV}$ and the effective ionization cost factor to $\gamma_{i}=3$. The ionization rate is approximated as a function of the total energy of electrons by the empirical formula

$$
\beta=\beta_{0}\left(\frac{\mathcal{E}}{\epsilon_{i}}\right)^{1 / 4} \exp \left(-\frac{2 \epsilon_{i}}{\mathcal{E}}\right)
$$

with $\mathcal{E} \equiv \frac{3}{2} T_{e}+\frac{1}{2} m_{e}\left(v_{e x}^{2}+v_{e \theta}^{2}\right)$ and $\beta_{0}=1.8 \times 10^{-13} \mathrm{~m}^{3} \mathrm{~s}^{-1}$, which reasonably fits experimental data [28] assuming a shifted Maxwellian distribution with mean velocity components $\left(v_{e x}, v_{e \vartheta}\right)$.

The working gas is xenon, injected with mass flow rate $\dot{M}=5 \mathrm{mg} \mathrm{s}^{-1}$ and velocity $V=200 \mathrm{~m} \mathrm{~s}^{-1}$. The applied voltage in all simulations is $U=220 \mathrm{~V}$. The profile of the applied radial magnetic field is given by a Gaussian curve,

$$
B(x)=B_{\max } \exp \left[-\left(\frac{x-\ell_{c}}{\ell_{B}}\right)^{2}\right]
$$

with $\ell_{B}=1.25 \mathrm{~cm}$ and $B_{\max }=22 \mathrm{mT}$ in the nominal case. The virtual cathode plane is located beyond the channel exit at a distance $\ell=3.5 \mathrm{~cm}$ from the anode. The temperature of electrons in the cathode plane is set to $\left.T_{e}\right|_{x=\ell}=5 \mathrm{eV}$.

\section{APPENDIX B: NUMERICAL METHODS}

For the time-dependent model, Eqs. (25) and (12) for neutrals and electron temperature are integrated independently from other equations, with time-explicit upwind schemes. The remaining transport equations for charge species (9)-(11) form a conservative hyperbolic system solved with the Harten-Lax-van Leer (HLL) scheme [29], using the velocity bounds proposed by Davis [30]. Time integration is explicit, except for the source term of Eq. (6) where the linear dependence on $I$ is accounted for with an implicit method to ensure stability.

The implementation of the low-frequency model is less straightforward and deserves some comments. Its workings are illustrated with a first-order explicit time-marching scheme as follows:

(i) $I$ and $N$ are known at $t_{0} ; \mathrm{n}, \beta$, and $\nu_{i w}$ are determined at $t_{0}$ by solving the quasisteady system for charged particles (18)-(22) and (14)-(16), and $\nu_{I}$ is simultaneously determined by satisfying Eq. (23);

(ii) the source term for neutrals is computed using the quantities determined at step (i) and the value of $I$ at $t_{0}$;

(iii) $N$ is advanced from $t_{0}$ to $t_{1}=t_{0}+d t$ using Eqs. (25) and (26) with the source term computed at step (ii);

(iv) $I$ is advanced from $t_{0}$ to $t_{1}$ using the value $\nu_{I}$ $\equiv d \ln I / d t$ computed at step (i); and 
(v) return to step (i) with the updated $I$ and $N$.

Note, however, that a second-order predictor-corrector time-marching scheme is actually used in simulations. The quasisteady solution of step (i) is determined using the same solver as the time-dependent model, applied to a timedependent generalization of Eqs. (19)-(22). The convergence to the quasisteady state is forced by a combination of underrelaxation for the electron energy equation (aimed at damping transit-time oscillations) and proportional-integral control for Eq. (23), whereas $\nu_{I}$ is the process variable and $U$ is the set point of the system.

For both models, the spatial domain is discretized into 81 nodes. The time step is adaptively determined so as to always satisfy the Courant-Friedrichs-Lewy condition (for charged species in the case of the TD model and for neutrals in the case of the LF model).

\section{APPENDIX C: ASYMPTOTIC LINEAR MODEL}

Let us assume small harmonic perturbations of $N$ and $I$ around the steady state,

$$
\begin{gathered}
N(x, t)=\bar{N}(x)+\hat{N}(x) \exp (J \omega t), \\
I(t)=\bar{I}+\hat{I} \exp (J \omega t) .
\end{gathered}
$$

The model (28)-(30) can be subsequently linearized as

$$
\begin{aligned}
& J \omega \hat{N}+V \frac{d \hat{N}}{d x}=-\bar{S} \hat{I}-\bar{I}\left[\bar{S}_{\Downarrow J \omega_{\bar{I}}^{-}}+\overline{\mathcal{S}}_{N} \hat{N}\right], \\
& \bar{u}_{\Downarrow J} \frac{\hat{I}}{\bar{I}}+\overline{\mathcal{U}}_{N} \hat{N}=0, \\
& \hat{N}(0)=0,
\end{aligned}
$$

where $\bar{S} \equiv \mathcal{S}(0, \bar{N})$ is a function of $x$ that corresponds to the value of $\mathcal{S}$ in the steady state. $\bar{S}_{v}$ is a function of $x$ that arises from the Fréchet differentiation of operator $\mathcal{S}$ with respect to $\nu_{I}$. Likewise, $\overline{\mathcal{S}}_{N}$ is a linear operator obtained by differentiation of $\mathcal{S}$ with respect to $N, \bar{u}_{\nu}$ is a scalar that stands for the derivative of $\mathcal{U}$ with respect to $\nu_{I}=d \ln I / d t$, and $\overline{\mathcal{U}}_{N}$ is a linear functional obtained by differentiation of $\mathcal{U}$ with respect to $N$. Defining operator $\overline{\mathcal{K}} \equiv \bar{I} \overline{\mathcal{S}}_{N}-\overline{I S}_{\nu} \bar{u}_{v}^{1} \overline{\mathcal{U}}_{N}$ and functional $\overline{\mathcal{G}} \equiv-\bar{u}_{\nu}{ }^{1} \overline{\mathcal{U}}_{N}$, Eqs. (C3) and (C4) can be rewritten in the simpler form

$$
\begin{gathered}
J \omega \hat{N}+V \frac{\partial \hat{N}}{\partial x}=-\gamma \bar{N} \frac{\hat{I}}{\bar{I}}-\overline{\mathcal{K}} \hat{N}, \\
J \omega_{\bar{I}}^{-}=\overline{\mathcal{G}} \hat{N},
\end{gathered}
$$

where $\gamma \equiv \overline{S I} / \bar{N}$ simply stands for the steady-state effective ionization frequency. Part of the mathematical abstraction associated with the linear model can be lifted using the
Schwartz kernel representation for operator $\overline{\mathcal{K}}$ and the Riesz representation for functional $\overline{\mathcal{G}}$,

$$
\begin{gathered}
{[\overline{\mathcal{K}} \hat{N}](x)=\int_{0}^{\ell} \bar{k}\left(x, x^{\prime}\right) \hat{N}\left(x^{\prime}\right) d x^{\prime},} \\
\overline{\mathcal{G}} \hat{N}=\int_{0}^{\ell} \Psi(x) \frac{\hat{N}(x)}{\bar{N}(x)} d x,
\end{gathered}
$$

which carries the idea that $\overline{\mathcal{K}}$ and $\overline{\mathcal{G}}$ are merely weighting functions for $\hat{N}$ and specified in terms of uniquely defined distributions $\bar{k}$ and $\Psi / \bar{N}$.

Proceeding now to the nondimensionalization of the linear system, it appears reasonable to assume that a perturbation of $N$ will introduce relative perturbations on the order of $\hat{N} / \bar{N}$ on the quasisteady state of charged particles, meaning in particular that

$$
\frac{1}{\bar{S}} \overline{\mathcal{S}}_{N} \hat{N}=\mathcal{O}\left(\frac{\hat{N}}{\bar{N}}\right), \quad \frac{1}{\bar{U}} \overline{\mathcal{U}}_{N} \hat{N}=\mathcal{O}\left(\frac{\hat{N}}{\bar{N}}\right) .
$$

In order to estimate the response of the quasisteady state of charged particles to oscillations of $I$, let us observe that electron-density conservation equation $D n / D t=I S$ can be expressed in terms of the scaled plasma density $\mathrm{n} \propto n / I$ as

$$
\frac{1}{S} \frac{D \mathrm{n}}{D t} \propto 1-\frac{n}{I S} \frac{1}{I} \frac{d I}{d t},
$$

where $D / D t$ stands for the convective derivative. Since the left-hand side of Eq. (C11) is a function of $S$ and $n$ which are both quasisteady quantities, the second term in the right-hand side makes it apparent that small oscillations of $I$ introduce relative perturbations on the order of $(\bar{n} / \overline{I S}) \times(J \omega \hat{I} / \bar{I})$ on the quasisteady state variables for charged species, meaning in particular that

$$
\frac{\bar{u}_{v}}{\bar{U}}=\mathcal{O}\left(\frac{\bar{n}}{\overline{I S}}\right), \quad \frac{\bar{S}_{v}}{\bar{S}}=\mathcal{O}\left(\frac{\bar{n}}{\overline{I S}}\right) .
$$

Having in mind that $\overline{I S}=-V d \bar{N} / d x$ and making use of Eqs. (C10) and (C12), the following nondimensional quantities of order unity are introduced:

$$
\begin{gathered}
x^{*} \equiv \frac{x}{\ell}, \\
\gamma^{*}\left(x^{*}\right)=\frac{\ell}{V} \gamma(x), \\
\Psi^{*}\left(x^{*}\right) \equiv \frac{\ell^{2} n_{0}}{V N_{0}} \Psi(x), \\
\bar{k}^{*}\left(x^{*}, x^{* \prime}\right) \equiv \frac{\ell^{2}}{V} \bar{k}\left(x, x^{\prime}\right),
\end{gathered}
$$

where $n_{0}$ and $N_{0}$ are characteristic densities for the plasma and for neutrals. The frequency and the state variables are 
made nondimensional irrespective of their actual orders of magnitude as follows:

$$
\begin{gathered}
\omega^{*} \equiv \frac{\ell}{V} \omega, \\
N^{*}\left(x^{*}\right) \equiv \frac{N(x)}{N_{0}}, \\
I^{*} \equiv \frac{I}{\bar{I}} .
\end{gathered}
$$

The linear breathing model is now expressed in a nondimensional form,

$$
\begin{gathered}
J \omega^{*} \hat{N}^{*}+\frac{\partial \hat{N}^{*}}{\partial x^{*}}=-\gamma^{*} \bar{N}^{*} \hat{I}^{*}-\overline{\mathcal{K}}^{*} \hat{N}^{*}, \\
J \omega^{*} \hat{I}^{*}=\frac{1}{\varepsilon} \int_{0}^{1} \Psi^{*} \frac{\hat{N}^{*}}{\bar{N}^{*}} d x^{*}, \\
\hat{N}^{*}(0)=0,
\end{gathered}
$$

which highlights the presence of a small parameter

$$
\varepsilon \equiv \frac{n_{0}}{N_{0}} \ll 1
$$

Turning now to the solution of the linearized model, we shall admit a priori the orderings $\operatorname{Re}\left(\omega^{*}\right) \gg 1$ and $\operatorname{Im}\left(\omega^{*}\right)=\mathcal{O}(1)$; their consistency with the solution arrived at is eventually verified. The exact solution of Eqs. (C13) and (C15) is of the form

$$
\begin{aligned}
\hat{N}^{*}\left(x^{*}\right)= & -\int_{0}^{x^{*}}\left\{\gamma^{*}\left(x^{* \prime}\right) \bar{N}^{*}\left(x^{* \prime}\right) \hat{I}^{*}+\left[\overline{\mathcal{K}}^{*} \hat{N}^{*}\right]\left(x^{* \prime}\right)\right\} \\
& \times \exp \left[J \omega^{*}\left(x^{* \prime}-x^{*}\right)\right] d x^{* \prime},
\end{aligned}
$$

which can be integrated by parts as

$$
\begin{aligned}
\hat{N}^{*}\left(x^{*}\right)= & J \hat{I}^{*} \omega^{*-1}\left[\gamma^{*}(x) \bar{N}^{*}(x)-\gamma^{*}(0) \bar{N}^{*}(0) \exp \left(-J \omega^{*} x^{*}\right)\right] \\
& +J \omega^{*-1}\left\{\left[\overline{\mathcal{K}}^{*} \hat{N}^{*}\right]\left(x^{*}\right)-\left[\overline{\mathcal{K}}^{*} \hat{N}^{*}\right](0) \exp \left(-J \omega^{*} x^{*}\right)\right\} \\
& +\hat{I}^{*} \mathcal{O}\left(\omega^{*-2}\right)+\hat{N}^{*} \mathcal{O}\left(\omega^{*-2}\right) .
\end{aligned}
$$

Remembering that $\bar{k}^{*}$ is of order unity and assuming that it is a sufficiently well-behaved function of $x^{*}$ and $x^{* \prime}$, it can be stated that $\overline{\mathcal{K}}^{*} \hat{N}^{*}$ is of order $\hat{N}^{*}$. Therefore, $\hat{N}^{*}$ is $\mathcal{O}\left(\hat{I}^{*} \omega^{*-1}\right)$ at the leading order,

$$
\begin{aligned}
\hat{N}^{*}\left(x^{*}\right)= & J \hat{I}^{*} \omega^{*-1}\left[\gamma^{*}(x) \bar{N}^{*}(x)-\gamma^{*}(0) \bar{N}^{*}(0) \exp \left(-J \omega^{*} x^{*}\right)\right] \\
& +\hat{I}^{*} \mathcal{O}\left(\omega^{*-2}\right) .
\end{aligned}
$$

The perturbation of neutral density consists thus of a shortwavelength oscillatory wave $\left[\right.$ term in $\left.\exp \left(-J \omega^{*} x^{*}\right)\right]$, superimposed on a standing wave. Injecting Eq. (C19) into Eq. (C14) and provided that $\Psi^{*}$ is a sufficiently well-behaved function of $x^{*}$, the integral of the short-wave oscillatory component becomes a residual of order $\mathcal{O}\left(\omega^{*-1}\right)$ and $\omega^{*}$ satisfies

$$
\omega^{* 2}=\frac{1}{\varepsilon} \int_{0}^{1} \gamma^{*}\left(x^{*}\right) \Psi^{*}\left(x^{*}\right) d x^{*}+\frac{1}{\varepsilon} \mathcal{O}\left(\omega^{*-1}\right)+\mathcal{O}(1) .
$$

Since $\gamma^{*}$ and $\Psi^{*}$ are real-valued functions of order unity, the above relation implies that $\operatorname{Re}\left(\omega^{*}\right) \propto \varepsilon^{-112} \gg 1$ and $\operatorname{Im}\left(\omega^{*}\right)$ $\leq \mathcal{O}(1)$, thus confirming the premises on which the approximation of $\hat{N}^{*}$ was derived. A corollary of this analysis is that operator $\overline{\mathcal{K}}$ can be neglected in Eq. (C6) if only the leadingorder expansions of $\omega$ and $\hat{N}$ are sought.
[1] E. Y. Choueiri, Phys. Plasmas 8, 1411 (2001).

[2] G. N. Tilinin, Sov. Phys. Tech. Phys. 22, 974 (1977).

[3] V. I. Baranov, Y. S. Nazarenko, V. A. Petrosov, A. I. Vasin, and Y. M. Yashnov, Proceedings of the 32nd AIAA Joint Propulsion Conference, Lake Buena Vista, FL (American Institute of Aeronautics and Astronautics, Washington, DC, 1996), pp. 963192.

[4] J. M. Fife, M. Martínez-Sánchez, and J. Szabo, Proceedings of the 33rd AIAA Joint Propulsion Conference, Seattle, WA (American Institute of Aeronautics and Astronautics, Washington, DC, 1997), pp. 97-3051.

[5] J.-P. Boeuf and L. Garrigues, J. Appl. Phys. 84, 3541 (1998).

[6] N. Yamamoto, K. Komurasaki, and Y. Arakawa, J. Propul. Power 21, 870 (2005).

[7] S. Chable and F. Rogier, Phys. Plasmas 12, 033504 (2005).

[8] J. M. Fife, Ph.D. thesis, Massachusetts Institute of Technology, 1998 (unpublished).
[9] L. Garrigues, A. Héron, J.-C. Adam, and J.-P. Boeuf, Plasma Sources Sci. Technol. 9, 219 (2000).

[10] S. Barral, K. Makowski, Z. Peradzyński, N. Gascon, and M. Dudeck, Phys. Plasmas 10, 4137 (2003).

[11] J. Bareilles, G. J. M. Hagelaar, L. Garrigues, C. Boniface, and J. P. Boeuf, Phys. Plasmas 11, 3035 (2004).

[12] F. I. Parra, E. Ahedo, J. M. Fife, and M. Martínez-Sánchez, J. Appl. Phys. 100, 023304 (2006).

[13] M. K. Scharfe, N. Gascon, M. A. Cappelli, and E. Fernandez, Phys. Plasmas 13, 083505 (2006).

[14] L. Albarède, S. Mazouffre, A. Bouchoule, and M. Dudeck, Phys. Plasmas 13, 063505 (2006).

[15] J. C. Adam, A. Héron, and G. Laval, Phys. Plasmas 11, 295 (2004).

[16] E. Ahedo, J. M. Gallardo, and M. Martínez-Sánchez, Phys. Plasmas 10, 3397 (2003).

[17] E. Ahedo, Phys. Plasmas 9, 3178 (2002). 
[18] S. Barral, Ph.D. thesis, Institute of Fundamental Technological Research of Warsaw, 2003.

[19] A. Bouchoule et al., Plasma Sources Sci. Technol. 10, 364 (2001).

[20] J.-P. Boeuf and L. Garrigues, Proceedings of the 26th International Electric Propulsion Conference, Kitakyushu, Japan (The Electric Rocket Propulsion Society, Worthington, OH, 1999), pp. 99-098.

[21] S. Barral, J. Miedzik, and E. Ahedo, Proceedings of the 42nd AIAA Joint Propulsion Conference, Sacramento, CA (American Institute of Aeronautics and Astronautics, Washington, D.C., 2006), pp. 2008-4632.

[22] D. Yu, C. Wang, L. Wei, C. Gao, and G. Yu, Phys. Plasmas 15,
113503 (2008)

[23] S. Barral, K. Makowski, Z. Peradzyński, and M. Dudeck, Phys. Plasmas 12, 073504 (2005).

[24] E. Fernandez, M. K. Scharfe, C. A. Thomas, N. Gascon, and M. A. Cappelli, Phys. Plasmas 15, 012102 (2008).

[25] S. Barral and Z. Peradzyński (unpublished).

[26] E. Ahedo, Phys. Plasmas 9, 4340 (2002).

[27] G. D. Hobbs and J. A. Wesson, Plasma Phys. 9, 85 (1967).

[28] J. A. Syage, Phys. Rev. A 46, 5666 (1992).

[29] A. Harten, P. D. Lax, and B. van Leer, SIAM Rev. 25, 35 (1983).

[30] S. F. Davis, SIAM (Soc. Ind. Appl. Math.) J. Sci. Stat. Comput. 9, 445 (1988). 